\title{
Behinderung, Inklusion, Teilhabe
}

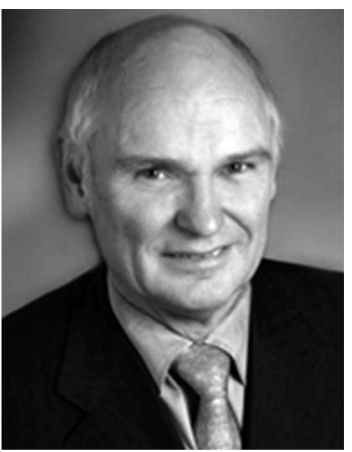

Dr. med. Gert von Mittelstaedt Präsident der Deutschen Gesellschaft für Sozialmedizin und Prävention (DGSMP)

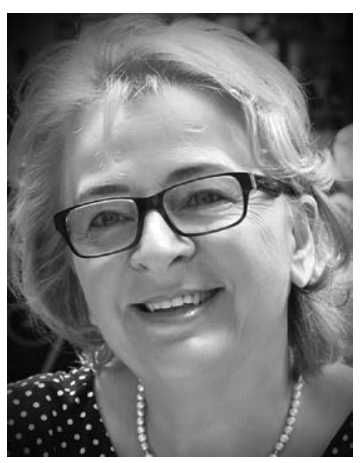

Dr. med. Sabine Grotkamp Mitglied des Vorstands (DGSMP) für den Fachbereich „Praktische Sozialmedizin und Rehabilitation" und die Arbeitsgruppe „ICF“

Bibliografie

DOI http://dx.doi.org/

10.1055/s-0042-111284

Gesundheitswesen 2016;

78: 424-425

(c) Georg Thieme Verlag KC

Stuttgart · New York

ISSN 0941-3790

Korrespondenzadresse

Dr. med. Gert von

Mittelstaedt

Medizinische Hochschule Hannover

Institut für Epidemiologie

Sozialmedizin und

Gesundheitssystemforschung

OE 5410

Carl-Neuberg-Straße 1

30625 Hannover

gertvm@gmx.de
Das Übereinkommen der Vereinten Nationen über die Rechte von Menschen mit Behinderungen (UN-Behindertenrechtskonvention - UNBRK) ist seit dem 26. März 2009 auch in Deutschland geltendes Recht. Das Leitbild ist Inklusion: Inklusion durch die breite Akzeptanz individueller Vielfalt in einer sich öffnenden Gesellschaft, welche den Wert jedes Einzelnen Menschen mit seinen Fähigkeiten und individuellen Voraussetzungen erkennt und anerkennt. „Nicht ohne uns über uns“ - die aktive Teilhabe von Menschen mit Behinderung auch an der Ausgestaltung der gesellschaftlichen Maßnahmen in der Umsetzung der UN-BRK ist eine wichtige Richtschnur der Behindertenpolitik in Deutschland. Der Ausschuss für die Rechte von Menschen mit Behinderungen bei den Vereinten Nationen hatte dafür der Bundesrepublik „Abschließende Bemerkungen über den ersten Staatenbericht Deutschlands“ vom 13. Mai 2015 mit auf den Weg gegeben. Darin enthalten waren u.a. die Forderungen nach einer Überarbeitung der gesetzlichen Definition von Behinderung, um sie mit den allgemeinen Grundsätzen und Bestimmungen der UN-BRK in Einklang bringen, nach ausreichenden Finanzmitteln, um De-Institutionalisierung und ein selbstbestimmtes Leben zu fördern, nach einem inklusiven Arbeitsmarkt und nach den notwendigen sozialen Diensten, um Inklusion, Selbstbestimmung und Teilhabe am Gemeinschaftsleben zu ermöglichen.

Ein wichtiger weiterer Schritt auf diesem Weg ist der aktuell vorliegende Referentenentwurf für ein Bundesteilhabegesetz (BTHG). In einem Brief an das Bundesministerium für Arbeit und Soziales (BAMS) zu diesem Referentenentwurf befürwortet die Deutsche Gesellschaft für Sozialmedizin und Prävention (DGSMP) ausdrücklich die Richtungsänderung hin zu einer personenzentrierten Sichtweise: „Die Leistungen sollen sich am persönlichen Bedarf orientieren und entsprechend eines bundeseinheitlichen Verfahrens personenbezogen ermittelt werden." Insbesondere wird begrüßt, dass in §99 SGB IX auf die Internationale Klassifikation der Funktionsfähigkeit, Behinderung und Gesundheit (ICF) und damit auf das bio-psycho-soziale Modell der Weltgesundheitsorganisation (WHO) Bezug genommen werden soll.

Die DGSMP und der Fachbereich „Praktische Sozialmedizin und Rehabilitation" sowie die Arbeitsgruppe ICF regen mit dem Schreiben an das BMAS im Referentenentwurf des Bundesteilhabegesetzes zu §99 SGB IX Leistungsberechtigter Personenkreis in Kapitel 2, Grundsätze der
Leistungen aus fachlicher Sicht folgende Überarbeitungen bzw. Ergänzungen an:

1. Die vorgenommene Definition der erheblichen Teilhabeeinschränkung entspricht nicht dem modernen Verständnis von Behinderung, wie es in der ICF zum Ausdruck kommt.

2. Sie ist nicht geeignet, die Schwere von Behinderung für die Betroffenen angemessen festzustellen.

3. Die vorgesehene Definition des leistungsberechtigten Personenkreises kann den Leistungszugang nicht sinnvoll steuern.

4. Die Quantifizierung von Beeinträchtigungen setzt ein Instrumentarium voraus, das sich idealerweise an der ICF orientiert, die ICF selbst ist hierzu nicht geeignet.

Die Forderung, dass Aktivitäten, die nicht ohne personelle oder technische Unterstützung möglich sind, in einer Mindestanzahl von Lebensbereichen auftreten müssen, widerspricht fundamental dem Verständnis von Behinderung, das der ICF und dem bio-psycho-sozialen Modell der WHO zugrunde liegt. Unter Behinderung sind die negativen Auswirkungen eines Gesundheitsproblems einer Person in Form von Schädigungen der Körperfunktionen und -strukturen und Beeinträchtigungen der Aktivitäten sowie der Teilhabe zu verstehen; diese können untereinander in Wechselwirkung stehen und durch den individuellen Lebenshintergrund (Umwelt- und personenbezogene Faktoren wie Alter, Geschlecht, Gewohnheiten, Bewältigungsstile) moduliert werden. Behinderung ist daher stets individuell und umfassend zu betrachten - sie kann nicht schematisch durch die Anzahl von Lebensbereichen mit Beeinträchtigung bewertet werden. Es gibt zudem keine Gleichwertigkeit aller Lebensbereiche, nicht zuletzt deshalb, weil sie nicht losgelöst voneinander zu sehen sind, sondern sich in Teilen auch überlappen.

Die bloße Anzahl von Lebensbereichen, in denen die Teilhabe eingeschränkt ist, bildet die individuelle Schwere der Behinderung nicht ab. Hierfür ist die Qualität, nicht die Quantität der Beeinträchtigungen ausschlaggebend. Es gilt dabei auch die relevanten möglichen Wechselwirkungen zwischen der Behinderung bzw. Funktionsfähigkeit und den individuellen Kontextfaktoren zu berücksichtigen, denn der reale Grad einer Beeinträchtigung der Teilhabe hängt nicht zuletzt auch vom Einfluss der Kontextfaktoren ab. Die im Referentenentwurf als Schwelle für den Zugang zu Leistungen der Eingliederungshilfe vorgesehene Anzahl von Lebensbereichen (mindestens 5 von 9) ist nicht wissenschaftlich 
begründet. Sie erscheint willkürlich festgesetzt, sodass eine solche Definition des Leistungsberechtigten Personenkreises weder praktikabel noch allgemein akzeptiert sein wird. Es besteht die Gefahr, dass in der Praxis Checklisten „abgehakt“ werden, anstelle der erforderlichen individuell umfassenden Sachverhaltsklärung.

Die Schwere einer (drohenden) Behinderung kann sachgerecht nur durch eine individuelle und umfassende Begutachtung/Bedarfserhebung auf der Grundlage des bio-psycho-sozialen Modells der WHO festgestellt werden. Daher ist die Nutzung der ICF durchgängig im Bedarfsfeststellungsverfahren, bei der Teilhabeplanung und bei Teilhabekonferenzen zu fordern.

Während an die erhebliche Teilhabeeinschränkung in § 99 Abs. 1 Satz 2 sehr hohe Anforderungen gestellt werden, bleibt die drohende erhebliche Teilhabeeinschränkung unbestimmt („nach fachlicher Kenntnis“). Die drohende erhebliche Teilhabeeinschränkung begründet aber nach $\S 99$ Abs. 1 Satz 3 denselben Leistungsanspruch wie die bereits eingetretene erhebliche Teilhabeeinschränkung. Damit entfaltet die Beschreibung des leistungsberechtigten Personenkreises in Satz 2 keine Steuerungswirkung bezüglich der Leistungsansprüche.
Die ICF wird für eine qualitative Bewertung von Beeinträchtigungen/Unterstützungsbedarfen im Sinne einer vereinfachenden Mengenbildung in unzulässiger Weise herangezogen und damit selbst als Instrument genutzt. Die ICF ist aber kein Assessment und kein Erhebungsinstrument, sondern ein Klassifikationssystem. Die ICF bietet für die sozialmedizinische Perspektive ein passendes Modell, indem sie die Auswirkungen von Gesundheitsstörungen differenziert betrachten lässt und indem sie die Einflussfaktoren des persönlichen Lebenshintergrundes berücksichtigen lässt. Beeinträchtigte Teilhabebereiche können mithilfe der ICF beschrieben bzw. klassifiziert werden; für eine Quantifizierung der jeweiligen Beeinträchtigungen bedarf es aber eines entsprechend geeigneten Instrumentariums. Hierzu werden in der ICF ansatzweise Beurteilungsmerkmale vorgeschlagen, die aber einer weiteren Ausdifferenzierung bedürfen. Die DGSMP mit ihrem Fachbereich „Praktische Sozialmedizin und Rehabilitation“ und der Arbeitsgruppe „ICF“ plädiert sowohl aus diesem Anlass als auch im Hinblick auf die Einheit der Sozialmedizin für eine durchgängige Orientierung - neben dem SGB IX - auch an der ICF und hofft, dass sie mit ihren Anregungen die Gesetzgebung zu diesem sehr zu begrüßenden Vorhaben konstruktiv unterstützen kann. 